\title{
XXXII. On the views of the Astronomer Royal respecting the modification of sounds by distance of propagation
}

\author{
Rev. J. Challis M.A. F.R.S. F.R.A.S.
}

To cite this article: Rev. J. Challis M.A. F.R.S. F.R.A.S. (1849) XXXII. On the views of the Astronomer Royal respecting the modification of sounds by distance of propagation , Philosophical Magazine Series 3, 35:236, 241-243, DOI: 10.1080/14786444908646345

To link to this article: http://dx.doi.org/10.1080/14786444908646345

曲 Published online: 30 Apr 2009.

Submit your article to this journal $\pi$

ЏII Article views: 2

Q View related articles $₫$ 
THE

LONDON, EDINBURGH AND DUBLIN

PHILOSOPHICAL MAGAZINE

A N D

JOURNAL OF SCIENCE.

\section{[THIRD SERIES.]}

OCTOBER 184.9.

XXXII. On the Vieves of the Astronomer Royal respecting the Modification of Sounds by Distance of Propagation. By the Rev. J. Chaldis, M.A., F.R.S., F.R.A.S., Plumian Professor of Astronomy and Experimental Philosophy in the University of Cambridge*.

T $N$ the Number of the Philosophical Magazine for last June, 1 the Astronomer Royal has stated it to be his belief that a musical sound may degenerate into a hiss, a buzz, or a whisper, and possibly into a roar, by mere distance of transmission. So singular and novel a theory can hardly be expected to pass current without being questioned. I consider myself entitled to call it in question, because a short while before it was promulgated I had proved in this Magazine, by what I conceive to be strict mathematical reasoning, that musical sounds do not by propagation become unmusical. The views of the Astronomer Royal profess to be only conjectures, being neither supported by mathematical reasoning, nor borne out by any experiment, and on this account they cannot well be brought under discussion. They are, however, based on a piece of mathematical reasoning which the Astronomer Royal has adopted from Mr. Stokes, and which admits of being discussed. Now I am prepared to show that this piece of reasoning is faulty in its logic, and that it consequently affords no pretext for the conjectures of the Astronomer Royal.

The rule of logic against which Mr. Stokes has offended may be stated as follows:- When the equations applicable to a proposed problem have been formed according to the principles of the department of science to which the problem belongs, and a solution of the equations has been obtained, it is not allowable to accept the solution in part and reject it in part : it is either wholly applicable or not at all. In the

* Communicated by the Author.

Phil. Mag. S. 3. Vol. 35. No. 236. Oct. 1849. 
instance of the hydrodynamical problem of the propagation of sound in plane-waves, Mr. Stokes has violated the above rule by accepting the analytical solution for certain values of the co-ordinates and the time, and rejecting it for other values. I proceed to make this assertion good.

The following is the enunciation of the problem. Assuming that a medium in which the pressure $p$ and density $p$ are related to each other by the equation $p=a^{2} \rho$, is capable of propagating waves in such a manner that the velocity and density of the particles of the medium are functions of the distance from a given plane, it is required to determine the motion.

Let $v$ be the velocity of any particle at the distance $z$ from the given plane, and at the time $t$ reckoned from a given epoch. Then by a known process, which it is unnecessary to go through, we have for the case of propagation in a single direction, the general solution,

$$
v=a . \text { Nap. } \log \rho=f(z-(a+v) t+c),
$$

and giving to the arbitrary function a form corresponding to a particular series of waves, we have the definite solution,

$$
v=a . \text { Nap. } \log \rho=m \sin \frac{2 \pi}{\lambda}(z-(a+v) t+c) .
$$

By these equations the velocity and density are given at all distances from the given plane at any instant, and the solution is in all respects complete. If, to ascertain the rate at which a given velocity and density are propagated, we differentiate the equations supposing $v$ and $\rho$ to be constant, it will be found that the velocity $v$ and corresponding density $e^{\frac{v}{a}}$ are propagated with the velocity $a+v$. The effect of the difference of rate of propagation of different parts of the wave presents itself as a continuous modification of the form of the wave. Mr. Stokes has exhibited this modification by a geometrical curve (see Phil. Mag. vol. xxxiii. p. 359), and in so doing has accepted the indications of analysis during an interval equal to $\frac{\lambda}{2 \pi m}$, but has rejected all its subsequent indications. To take $a$ numerical instance, let $a=916$ feet in a second, $m=1$ foot in a second, and $\lambda=10$ feet. Then it would be admitted in this instance, that during an interval of one second and six tenths, in which the wave travels over 14.59 feet, the analysis indicates truly, but not for any longer period. This procedure Mr. Airy has signified his approval of by saying, that "Mr. Stokes has shown that a distinct 
meaning can be given to the solution up to a certain point in the progress of the wave." If we may believe these two mathematicians, the wave at this point suddenly emancipates itself from the control of analysis, and the subsequent motion can be pursued by no other means than conjecture. Accordingly each has favoured us with his conjectures. Mr. Airy's are contained in the article which has given occasion to these remarks, and Mr. Stokes's in the communication already referred to (Phil. Mag. vol. xxxiii. pp. 352-356).

It is not my intention to enter into any consideration of these conjectures, which, in my opinion, can only be regarded as proofs that the authors of them have fallen into an erroneous course of reasoning. In any legitimate application of analytical calculation to a physical question, a spontaneous failure of the analysis, leaving no resource but conjecture, can never occur. Before such necessity has arisen, some fault in logic must have been committed. I do not doubt that I point out the fault committed in the case under discussion by saying, that certain consequences deduced by analysis from the premises are received, while other consequences equally deduced by analysis from the same premises are rejected. The analytical solution of the problem of plane-waves is, as I have already stated, that the velocity $v$ at any point of the wave and the corresponding density are propagated with the velocity $a+v$. In consequence of the different rate of propagation of different parts of the wave, two different states of the medium must occupy eventually the same position at the same time. For instance, a position of maximum velocity is a position of no velocity when the interval $t$ has become $\frac{\lambda}{4 m}$; that is, taking the numerical example before adduced, after the lapse of two seconds and a half, when the wave has travelled through 2290 feet. We are thus brought by the analysis to an absurdity. It is true the absurdity is first consummated at the end of the interval $\frac{\lambda}{2 \pi m}$. Mr. Stokes avails himself of this circumstance to limit his acceptance of the results of analysis to this interval, and avoids the consideration of the absurdity; whereas right reasoning demands, if in any case an absurdity is strictly deduced from premises, that all results from the same premises should be rejected. It is in vain to urge, as Mr. Airy does, that the absurdity indicates the transition of the waves from a musical to an unmusical condition. $A$ hiss and a roar are matters of experience and possible; they cannot therefore be symbolized by an impossibility. The only legitimate inference from the absurdity is, that the hypo- 
thesis of the problem is at fault; that it is not allowable to make the supposition of plane-roaves.

Mr. Airy's views, as I have said, are not borne out by any experiment. On the contrary, an experiment may be adduced which contradicts them. The possibility of hearing distinctly words spoken at a distance, depends on the faithfulness with which the air transmits the impressions made on it by the organs of voice. As the difference between the sound of one letter and that of another corresponds to a difference in the form of the curve representing the succession and magnitudes of the condensations impressed, it is necessary that that form should remain unchanged by distance of transmission in order that words heard at different distances may be the same sounds. The law of transmission expressed by the formula $a+v$, which is the basis of Mr. Airy's speculations, is opposed to this constancy of form. M. Biot, however, has recorded an experiment made at Paris, according to which, words pronounced at one end of a cylindrical tube 3120 feet in length, were perfectly distinct at the other end.

Cambridge Observatory, August 22, 1849.

XXXIII. An Investigation on the Chemical Nature of Wax. By Benjamin Collins Brodie, Esq.*

[Continued from vol. xxxiii. p. 391.]

III. $†$ On Myricine.

THAVE placed the investigation of the Chinese wax be1 tween that of the cerotic acid and of the residue of the bees'-wax which remains after that substance has been separated from it. By the saponification of this Chinese wax we procure, as I have shown, an acid identical with the cerotic acid from bees'-wax, and also the alcohol of this acid, so that the chemical history of these substances is closely connected. We have moreover in the Chinese wax to deal with a substance found in nature in a state of great purity, the products of the decomposition of which by alkalies and by heat can readily be prepared and examined. The knowledge of the relation of these products to one another throws great light upon the nature of myricine, which is not a pure substance, and the chemical relations of which are complex.

I have stated that the first extracts of wax with alcohol give with acetate of lead an abundant precipitate in a hot alcoholic

* From the Philosophical Transactions for 1849, part i.; having been received by the Royal Society May 11, and read November 23, 1848 .

+ Part I. was inserted in vol. xxxiii. p. 217, and Part II. at p. 378 of the same volume of this Journal. - Ep. 